VoL. 61 (2000) [319-327]

\title{
ARITHMETIC ON CERTAIN FAMILIES OF ELLIPTIC CURVES
}

\author{
Andrzej DąBrowski and Malgorzata Wieczorek
}

\begin{abstract}
Consider a family of elliptic curves $E_{(B)}: y^{2}=x^{3}+d_{0}^{2} A_{0} x+d_{0}^{3} B\left(A, A_{0}, d_{0}\right.$ fixed integers). We prove that, under certain conditions on $A_{0}$ and $d_{0}$, the rational torsion subgroup of $E_{(B)}$ is either cyclic of order $\leq 3$ or non-cyclic of order 4 . Also, assuming standard conjectures, we establish estimates for the order of the Tate-Shafarevich groups as $B$ varies.
\end{abstract}

\section{INTRODUCTION}

Let $E: y^{2}=x^{3}+A x+B \quad\left(A, B \in \mathbb{Z}, 4 A^{3}+27 B^{2} \neq 0\right)$ be a fixed elliptic curve over $\mathbb{Q}$. For each $d \neq 0$ let $E_{d}$ be the elliptic curve $E_{d}: y^{2}=x^{3}+d^{2} A x+d^{3} B$.

One can prove that for all but finitely many square-free integers $d \neq 0$, the torsion subgroup of $E_{d}(\mathbb{Q})$ is one of $(0), \mathbb{Z} / 2, \mathbb{Z} / 2 \oplus \mathbb{Z} / 2$, and a necessary condition that $E_{d}$ posseses $\mathbb{Q}$-rational point of order greater than 2 is $d \mid 4 A^{3}+27 B^{2}[10]$.

We shall prove that the $\mathbb{Q}$-torsion subgroup of $E_{(B)}: y^{2}=x^{3}+d_{0}^{2} A_{0} x+d_{0}^{3} B\left(A_{0}, d_{0}\right.$ fixed) is, under certain conditions on $A_{0}$ and $d_{0}$, one of $(0), \mathbb{Z} / 2, \mathbb{Z} / 3, \mathbb{Z} / 2 \oplus \mathbb{Z} / 2$ (Section 1). Let $N_{B}, R_{B}$, and $\amalg\left(E_{(B)}\right)$ denote respectively the conductor, the regulator, and the Tate-Shafarevich group (conjecturally finite) of $E_{(B)}$. Assuming the Birch and Swinnerton-Dyer conjecture for all $E_{(B)}$ we prove that

$$
N_{B}^{1 / 12-\varepsilon} \ll R_{B} \cdot \sharp \amalg\left(E_{(B)}\right) \ll N_{B}^{1 / 12+\varepsilon}
$$

for infinitely many $B$ (the lower bound is actually valid for all $B$ ) (Section 2). We also comment on the behaviour of the order of $\amalg$ in other families of elliptic curves. In section 3 we give some evidence for Mazur's conjecture on the variation of the rank in a family in a special case.

\section{TORSION POINTS ON CERTAIN FAMILIES OF ELLIPTIC CURVES}

1.1. Construction of Certain families of elliptic CuRVes. We start with the following well known result.

Received 15th July, 1999

Copyright Clearance Centre, Inc. Serial-fee code: 0004-9727/00 \$A2.00+0.00. 
Proposition 1.1.1. [12, Ex. 8.13(a), p.238] Let $E / \mathbb{Q}$ be an elliptic curve over $\mathbb{Q}$ with a rational torsion point of order $\geq 4$. Then $E$ has an equation of the form

$$
y^{2}+u x y+v y=x^{3}+v x^{2}
$$

with $u, v \in \mathbb{Q}$.

The family (1) can easily be rewritten into the following equivalent form:

$$
\begin{aligned}
E(u, v): \quad y^{2}= & x^{3}+\left(-\frac{1}{3} v^{2}+\left(-\frac{1}{6} u^{2}+\frac{1}{2} u\right) v-\frac{1}{48} u^{4}\right) x \\
& +\left(\frac{2}{27}\left(v+\frac{1}{4} u^{2}\right)^{3}-\frac{1}{6} u v\left(v+\frac{1}{4} u^{2}\right)+\frac{1}{4} v^{2}\right)
\end{aligned}
$$

Let $E=E(A, B): y^{2}=x^{3}+A x+B \quad\left(A, B \in \mathbb{Z}, 4 A^{3}+27 B^{2} \neq 0\right)$ be an elliptic curve. For each $0 \neq d \in \mathbb{Z}$ consider its quadratic twist

$$
E_{d}: y^{2}=x^{3}+d^{2} A x+d^{3} B
$$

$E_{d}$ has a rational point of order $\geq 4$ if and only if it is of the form (2) with some $u, v \in \mathbb{Q}$. In particular, we have

$$
16 v^{2}+\left(8 u^{2}-24 u\right) v+\left(u^{4}+48 A d^{2}\right)=0
$$

Now

$$
\Delta_{v}=\left(8 u^{2}-24 u\right)^{2}-4 \cdot 16 \cdot\left(u^{4}+48 A d^{2}\right)=2^{6}\left(-6 u^{3}+9 u^{2}-48 A d^{2}\right)
$$

Hence $\Delta_{v} \in \mathbb{Q}^{2}$ if and only if $-6 u^{3}+9 u^{2}-48 A d^{2}=y^{2}$ with certain $y \in \mathbb{Q}$ if and only if $E(A ; d): y^{2}=x^{3}-27 x-54\left(32 A d^{2}-1\right)$ has solution in $x, y \in \mathbb{Q}$.

Note that $E(A ; d)$ is an elliptic curve if and only if $A \neq 0$.

Proposition 1.1.2. Assume $A \neq 0$. Then $E(A ; d)(\mathbb{Q})=(0)$ implies that the torsion part $E(A, B)_{d}(\mathbb{Q})_{\text {tors }}$ is one of $(0), \mathbb{Z} / 2, \mathbb{Z} / 3, \mathbb{Z} / 2 \oplus \mathbb{Z} / 2$.

Proof: Combine the above construction with Mazur's theorem [9].

1.2. TORsion part of $E(A ; d)(\mathbb{Q})$. Assume that $E(\mathbb{Q})_{\text {tors }} \neq(0)$; then we know [9] that $E(\mathbb{Q})_{\text {tors }}$ contains only points of orders 2 or 3 or 5 or 7 .

(a) Non-existence of point of order 5 .

By [5] we know that $E(A ; d)(\mathbb{Q})$ contains a point of order 5 if and only if $E(A ; d)$ is of the form

$$
y^{2}+(1-c) x y-c y=x^{3}-c x^{2}, \quad \text { with some } c \in \mathbb{Q}
$$


which is equivalent to

$$
\begin{aligned}
y^{2}= & x^{3}+\left[-\frac{1}{3} c^{2}+\frac{1}{6} c(c-1)^{2}-\frac{1}{48}(c-1)^{4}+\frac{1}{2} c(c-1)\right] x \\
& +\left[\frac{2}{27}\left(-c+\frac{1}{4}(c-1)^{2}\right)^{3}-\frac{1}{6} c(c-1)\left(-c+\frac{1}{4}(c-1)^{2}\right)+\frac{1}{4} c^{2}\right]
\end{aligned}
$$

Comparing the coefficients we obtain

$$
-(c-1)^{4}+8 c(c-1)^{2}+8 c^{2}-24 c+2^{4} 3^{4}=0 .
$$

The above equation has no solution in $c \in \mathbb{Q}$, hence $E(A ; d)(\mathbb{Q})$ contains no points of order 5 .

(b) Non-existence of point of order 7.

We know [5] that $E(A ; d)(\mathbb{Q})$ contains a point of order 7 if and only if $E(A ; d)$ is of the form

$$
y^{2}+\left(1+t-t^{2}\right) x y+\left(t^{2}-t^{3}\right) y=x^{3}+\left(t^{2}-t^{3}\right) x^{2}, \quad \text { with some } t \in \mathbb{Q}
$$

which is equivalent to

$$
\begin{aligned}
y^{2}= & x^{3}+\left[-\frac{1}{3}\left(\frac{1}{4}\left(1+t-t^{2}\right)^{2}+\left(t^{2}-t^{3}\right)\right)^{2}+\frac{1}{2}\left(1+t-t^{2}\right)\left(t^{2}-t^{3}\right)\right] x \\
& +\left[\frac{2}{27}\left(\frac{1}{4}\left(1+t-t^{2}\right)^{2}+\left(t^{2}-t^{3}\right)\right)^{3}\right. \\
& \left.-\frac{1}{6}\left(\frac{1}{4}\left(1+t-t^{2}\right)^{2}+\left(t^{2}-t^{3}\right)\right)\left(1+t-t^{2}\right)\left(t^{2}-t^{3}\right)+\frac{1}{4}\left(t^{2}-t^{3}\right)^{2}\right]
\end{aligned}
$$

Comparing the coefficients we obtain

$$
\left(1+t-t^{2}\right)^{4}+8\left(1+t-t^{2}\right)^{2}\left(t^{2}-t^{3}\right)+16\left(t^{2}-t^{3}\right)^{2}-24\left(1+t-t^{2}\right)\left(t^{2}-t^{3}\right)-2^{4} 3^{4}=0 .
$$

The above equation has no integer (hence rational) solutions.

(c) Points of order 3 .

We have, similarly to [5], that $E(A ; d)(\mathbb{Q})$ contains a point of order 3 if and only if $E(A ; d)$ is of the form

$$
y^{2}+a x y+b y=x^{3} \quad(a, b \in \mathbb{Q})
$$

which is equivalent to

$$
\begin{aligned}
y^{2}= & x^{3}+\left(-\frac{1}{48} a^{4}+\frac{1}{2} a b\right) x \\
& +\left(\frac{1}{27 \cdot 32} a^{6}-\frac{1}{24} a^{3} b+\frac{1}{4} b^{3}\right) .
\end{aligned}
$$


Comparing the coefficients we obtain an equation in $a$ :

$$
a^{12}-2^{5} a^{9}-2^{4} 3^{5} a^{8}+2^{9} 3^{5} a^{5}+2^{8} 3^{9} a^{4}-2^{12} 3^{6}\left(1-2^{5} d^{2} A\right) a^{3}-2^{12} 3^{12}=0 .
$$

In the case $A=1$ one checks the above equation has no rational solutions.

(d) Points of order 2 .

$E(A ; d)(\mathbb{Q})$ contains a point of order 2 if and only if $x^{3}-27 x-54\left(32 A d^{2}-1\right)=0$ has solutions in $x \in \mathbb{Z}$. Certainly this is not the case for $A=1, d \equiv 2(\bmod 5)$.

1.3. FreE PART OF $E(A ; d)(\mathbb{Q})$. It is not difficult to calculate finite products of the type $f(x)=\prod_{p<x}\left(p^{-1} \sharp E(A ; d)\left(\mathbb{F}_{p}\right)\right), x$ big enough. One expects that the rank of $E(A ; d)(\mathbb{Q})$ is zero if $f(x)$ is bounded. To calculate the rank exactly one can use, say, (pseudo)algorithms described in Cremona's book [1], or an executable version of the program from his ftp server. For example: $\operatorname{rank} E(1 ; 2)(\mathbb{Q})=0, \operatorname{rank} E(1 ; 7)(\mathbb{Q})=1$.

1.4. An Example. Take $A_{0}=1, d_{0}=2$, and consider the family $E_{(B)}: y^{2}=$ $x^{3}+4 x+8 B$. Considering the reduction modulo 5, and applying Proposition 1.1 .2 and $[\mathbf{1 0}]$, we conclude that

$$
\begin{aligned}
& E_{(2 B)}(\mathbb{Q})_{\text {tors }} \subset \mathbb{Z} / 3 \text { for } B \equiv 2,3(\bmod 5), \\
& E_{(2 B)}(\mathbb{Q})_{\text {tors }} \subset \mathbb{Z} / 2 \oplus \mathbb{Z} / 2 \text { for } B \equiv 0,1,4(\bmod 5), \\
& E_{(2 B+1)}(\mathbb{Q})_{\text {tors }}=(0) \text { for } B \equiv 0,4(\bmod 5), \\
& E_{(2 B+1)}(\mathbb{Q})_{\text {tors }} \subset \mathbb{Z} / 2 \oplus \mathbb{Z} / 2 \text { for } B \equiv 1,2,3(\bmod 5) .
\end{aligned}
$$

Note however (by the Lutz-Nagell theorem) that $E_{(B)}(\mathbb{Q})$ contains a point of order 2 if and only if $B=k\left(k^{2}+1\right), k \in \mathbb{Z}$.

\section{BOUNDS ON THE SIZE OF THE ORDER OF THE TATE-SHAFAREVICH GROUP}

In this section we shall establish estimates for the orders of $\amalg\left(E_{(B)}\right)$ as $B$ varies (Theorem 2.4.1).

2.1. BOUNDS ON THE REAL PERIOD. Let $\pi_{\infty}(E)$ denote the real period of $E$.

LEMMA 2.2 .1 . We have $\pi_{\infty}\left(E\left(A_{0}, B\right)_{d_{0}}\right) \gg \ll B^{-\frac{1}{6}}$.

Proof: Note that

$$
\pi_{\infty}\left(E\left(A_{0}, B\right)_{d_{0}}\right)=d_{0}^{1 / 2} \pi_{\infty}\left(E\left(A_{0}, B\right)\right)
$$


Now

$$
\begin{gathered}
\pi_{\infty}\left(E\left(A_{0}, B\right)\right)=\int_{-\infty}^{\infty} \frac{d x}{\sqrt{x^{3}+A_{0} x+B}}=\int_{1}^{\infty}+\int_{-1}^{1}+\int_{-\infty}^{-1} \\
\int_{1}^{\infty} \frac{d x}{\sqrt{x^{3}+A_{0} x+B}} \ll \int_{1}^{\infty} \frac{d x}{\sqrt{x^{3}+B}} \ll \int_{1}^{\infty} \frac{d x}{\left(x+B^{1 / 3}\right)^{3 / 2}} \ll B^{-1 / 6} \\
\int_{-1}^{1} \frac{d x}{\sqrt{x^{3}+A_{0} x+B}} \ll \int_{-1}^{1} \frac{d x}{\sqrt{B}} \ll B^{-1 / 2} . \\
\int_{-\infty}^{-1} \frac{d x}{\sqrt{x^{3}+A_{0} x+B}}=\left(\int_{-\infty}^{-B^{1 / 3}}+\int_{-B^{1 / 3}}^{-1}\right) \frac{d x}{\sqrt{x^{3}+A_{0} x+B}} \ll B^{-1 / 6}
\end{gathered}
$$

The above proves the "«" part. For the " $\gg$ " part see [6, p.159].

2.2. BOUNDS ON THE CONDUCTOR. Let

$$
E\left(A_{0}, B\right)_{d_{0}}: \quad y^{2}=x^{3}+d_{0}^{2} A_{0}+d_{0}^{3} B
$$

We have

$$
\Delta=-2^{4}\left(4 d_{0}^{6} A_{0}^{3}+27 d_{0}^{6} B^{2}\right) \ll B^{2} .
$$

Hence $N_{B}=N_{E\left(A_{0}, B\right)_{d_{0}}} \ll B^{2}$.

Now assume $A_{0} d_{0} \neq 0$.

LEMMA 2.2.1. For infinitely many integers $B$ we have $N_{B} \gg B^{2}$.

PRoOF: Indeed, by Iwaniec's work [4] we know that the polynomial $27 x^{2}+a$ ( $a$ fixed odd integer) takes infinitely many values of the form $p_{1} p_{2},\left(p_{1}, p_{2}\right.$ different rational primes). The assertion follows.

2.3. AN UPPER BOUND FOR $\prod \pi_{p}$.

LEMMA 2.3.1. We have

$$
\prod_{p \mid N_{B}} \pi_{p}=O\left(B^{\varepsilon}\right)
$$

Proof: Take a rational prime $p \mid N_{B}$. We obtain, using Tate's algorithm [13], that the corresponding Kodaira symbol is never of type $I_{\nu}(\nu>0)$. Hence $\pi_{p} \leq 4$, and the assertion follows.

2.4. ESTIMATES ON THE ORDER OF Ш. 
TheOrem 2.4.1. Fix integers $A_{0}, d_{0}\left(A_{0} d_{0} \neq 0\right)$. Assume the Birch and Swinnerton-Dyer conjecture holds for all $E_{(B)}=E\left(A_{0}, B\right)_{d_{0}}$. Then

$$
R_{B} \cdot \sharp W\left(E_{(B)}\right) \gg N_{B}^{1 / 12-\varepsilon} \text {. }
$$

(b) Assume additionally the generalised Lindelof conjecture [3] for the family $E\left(A_{0}, B\right)_{d_{0}}$. Then there exist infinitely many $B$ such that

$$
R_{B} \cdot \sharp U I\left(E_{(B)}\right) \ll N_{B}^{1 / 12+\varepsilon} .
$$

Proof:

(a) Using the above bounds and the Birch and Swinnerton-Dyer conjecture for all $E\left(A_{0}, B\right)_{d_{0}}$, we have

$$
R_{B} \cdot \sharp \amalg\left(E_{(B)}\right) \gg B^{1 / 6-\varepsilon} \gg N_{B}^{1 / 12-\varepsilon} .
$$

(b) The generalised Lindelöf hypothesis for $L(E, s)$ implies $L^{(r)}(E, 1)=$ $O\left(N_{E}^{\epsilon}\right)$ (see [3, p.154]). On the other hand $\pi_{\infty}\left(E\left(A_{0}, B\right)_{d_{0}}\right) \ll B^{-1 / 6}$. Also we have $\sharp E\left(A_{0}, B\right)_{d_{0}}(\mathbb{Q})_{\text {tors }} \leq 16$ by [9], and $\prod_{p \mid N_{B}} c_{p} \geq 1$.

The above estimates and the Birch and Swinnerton-Dyer conjecture for all the $E\left(A_{0}, B\right)_{d_{0}}$ imply $R_{B} \cdot \sharp \amalg\left(E_{(B)}\right) \ll B^{1 / 6+\varepsilon}$. By Iwaniec's result we have $N_{B} \gg B^{2}$ for infinitely many $B$. The assertion follows.

2.5. REMARK. Assuming additionally the Lang conjecture $R_{E} \gg N_{E}^{-\varepsilon}$, one can state the result in the form:

$$
N_{B}^{1 / 12-\varepsilon} \ll \sharp \amalg\left(E_{(B)}\right) \ll N_{B}^{1 / 12+\varepsilon}
$$

for infinitely many $B$.

2.6. EXAMPLE. Consider the family $E(B): y^{2}=x^{3}+12 x+16 B,(B \in \mathbb{Z})$. Here $\Delta=2^{8} 3^{3}\left(1+B^{2}\right)$, and one checks that the reduction is split multiplicative at all primes $p \neq 2,3$. On the other hand, it is classical that every odd prime divisor of $1+B^{2}$ is of the type $4 k+1$. Hence, using [11, Propositions 1 and 3$]$ we conclude that for infinitely many $B$ 's the root number $\varepsilon(E(B))$ depends only on the local root numbers at 2 and 3. As a consequence we obtain that, at least conjecturally, the rank is even (maybe zero) for infinitely many $B$ 's.

\subsection{REMARKS.}

(i) Part (b) of the theorem is consistent with the following conjecture of Lang. Write $E: y^{2}=x^{3}+a x+b$, with $a, b \in \mathbb{Z} ;$ let $H(E)=\max \left(|a|^{3},|b|^{2}\right)$. Lang [6] conjectures that

$$
R_{E} \cdot \sharp \amalg(E) \ll H(E)^{1 / 12} N^{\varepsilon(N)} c^{r}(\log N)^{r},
$$


with some universal constant $c$, and $\varepsilon(N) \rightarrow 0$ as $N \rightarrow \infty$.

(ii) The case $A=0$. Note that $E(0 ; d)$ is not an elliptic curve. Hence the family $E(0, B)_{d_{0}}$ cannot be treated by our method. Such a family was studied from an analytic point of view in [7]; consequences for the order of III (in a case $E(d): x^{3}+y^{3}=d, d$ cubic-free) are as follows:

$$
N_{E(d)}^{1 / 6-\varepsilon} \ll \sharp \amalg(E(d)) \ll N_{E(d)}^{1 / 3+\varepsilon}
$$

for infinitely many $d$.

(iii) The family $E\left(A_{0} ; d\right)(d \in \mathbb{Z})$ also has interesting properties. Take, say, $A_{0}=1$, and denote $E(d)=E(1 ; d)$. One can show that for all $d \in \mathbb{Z}$

$$
N_{E(d)}^{1 / 12-\varepsilon} \ll \sharp \amalg(E(d)) \cdot R_{E(d)} \ll N_{E(d)}^{1 / 9+\varepsilon} .
$$

(iv) Consider the family $E(p): y^{2}=x^{3}+p x(p$ prime $\equiv 7,11(\bmod 16))$. Then $\operatorname{rank} E(p)(\mathbb{Q})=0[12, \mathrm{p} .311]$, and one can show (assuming the Birch and Swinnerton-Dyer conjecture) that

$$
N_{E(p)}^{1 / 8-\varepsilon} \ll \sharp \amalg(E(p)) \ll N_{E(p)}^{1 / 8+\varepsilon}
$$

for all such primes.

(v) Denote by $E_{d}$ ( $d$ square-free integer) the quadratic twist of $E$. Mai and Murty [8] proved, assuming the Birch and Swinnerton-Dyer conjecture for all $E_{d}$ 's, that there exist infinitely many $d$ such that $E_{d}$ has rank zero and

$$
\sharp \amalg\left(E_{d}\right) \gg N_{E_{d}}^{1 / 4-\varepsilon} \text {. }
$$

As for the upper bound, one can prove [2] (assuming additionally the generalised Lindelöf hypothesis) that for the same family $E_{d}$ (of rank zero) one has

$$
\sharp \mathrm{W}\left(E_{d}\right) \ll N_{E_{d}}^{1 / 4+\varepsilon} \text {. }
$$

2.8. Numerical observation. Let $E(d): y^{2}=x^{3}-d^{2} x$ ( $d \geq 1$ an odd square-free integer) denote the congruent number elliptic curve. Let

$$
\begin{aligned}
& \eta(z)=q^{1 / 24} \prod_{n=1}^{\infty}\left(1-q^{n}\right), \quad \Theta(z)=\sum_{n=-\infty}^{\infty} q^{n^{2}} \\
& f(z):=\eta(8 z) \eta(16 z) \Theta(2 z)=\sum_{n=1}^{\infty} a(n) q^{n}
\end{aligned}
$$


For $E(d)$ 's of rank zero (assuming the Birch and Swinnerton-Dyer conjecture) we have (see [14]):

$$
\sqrt{\sharp \amalg(E(d))}=\frac{|a(d)|}{\tau(d)},
$$

where $\tau(d)$ denotes the number of divisors of $d$.

We have tabulated all odd square-free $d$ 's $(d \leq 20000)$ such that $\operatorname{rank} E(d)=0$ and $\sharp \amalg(E(d))=1$. The calculations led us to the following observation. Let

$$
\begin{aligned}
& A:=\{d \in \mathbb{N}: d \text { odd square-free, rank } E(d)=0, \sharp \amalg(E(d))=1\}, \\
& B:=\{n \in \mathbb{N}: n \text { is a sum of digits of a certain } d \in A\} .
\end{aligned}
$$

CONJECTURE. Let $n$ be a positive integer. Then $n \in B$ if and only if $9 \nmid n$.

\section{Evidence for Mazur's CONJECture}

In this section we check Mazur's conjecture in a special case, which concerns the variation of $\operatorname{rank} E(1 ; t)(\mathbb{Q})$ with $t \in \mathbb{Q}$ : either there are only finitely many $t \in \mathbb{Q}$ such that the rank of $E(1 ; t)(\mathbb{Q})$ is positive, or else the set of all such $t$ is dense in $\mathbb{R}$.

First, it is plain that $(x, y)=(-2,5)$ is a point of infinite order on $E(1 ; 5 / 24): y^{2}=$ $x^{3}-27 x-21$. Also, $(-2,5 / 24)$ is a point of infinite order on $E: 54 \cdot 32 y^{2}=x^{3}-27 x+29$. Therefore, $E(\mathbb{Q}) \cap E(\mathbb{R})^{0}$ is dense in $E(\mathbb{R})^{0}$, and hence $E(\mathbb{Q})$ contains points of infinite order of the form $(q, k / l)$ with $q \in \mathbb{Q}, k, l \in \mathbb{Z},(k, l)=1$. Since the curves $E(1 ; t)$ have no rational torsion points for a dense set of $t \in \mathbb{Q} \subset \mathbb{R}$ (use Section 1.2), we obtain the following result.

THEOREM 3.1. The set of all rational $t$ such that $E(1, t)(\mathbb{Q})$ has positive rank is dense in $\mathbb{R}$.

QUESTION. It would be interesting to have any information concerning the behaviour of $\operatorname{rank} E\left(A_{0} ; d\right)(\mathbb{Q})$ as $d \in \mathbb{Z}$ varies. One possible way is to study the variation of the root number in such a family using ideas from [11].

\section{REFERENCES}

[1] J.E. Cremona, Algorithms for modular elliptic curves (Cambridge University Press, Cambridge, 1992).

[2] A. Dąbrowski and J. Pomykała, 'On the order of the Tate-Shafarevich group in a quadratic family of elliptic curves', (submitted).

[3] D. Goldfeld, J. Hoffstein and S.J. Patterson, 'On automorphic functions of half-integral weight with applications to elliptic curves', in Number Theory Related to Fermat's Last Theorem (Birkhäuser, Boston, MA, 1982), pp. 153-193. 
[4] H. Iwaniec, 'Almost-primes represented by quadratic polynomials', Invent. Math. 47 (1978), 171-188.

[5] D.S. Kubert, 'Universal bounds on the torsion of elliptic curves', Proc. London Math. Soc. 33 (1976), 193-237.

[6] S. Lang, 'Conjectured diophantine estimates on elliptic curves', in Arithmetic and Geometry - Papers dedicated to I.R. Shafarevich 1 (Birkhäuser, Boston, MA, 1983), pp. 155-171.

[7] D. Lieman, 'Nonvanishing of $L$-series associated to cubic twists of elliptic curves', Ann. Math. 140 (1994), 81-108.

[8] L. Mai and M.R. Murty, 'A note on quadratic twists of an elliptic curve', in CRM Proc. Lecture Notes 4 (American Mathematical Society, Providence, RI, 1994), pp. 121-124.

[9] B. Mazur, 'Rational isogenies of prime degree', Invent. Math. 44 (1978), 129-162.

[10] L.D. Olson, 'Torsion points on elliptic curves with given j-invariant', Manuscripta Math. 16 (1975), 145-150.

[11] D.E. Rohrlich, 'Variation of the root number in families of elliptic curves', Compositio Math. 87 (1993), 119-151.

[12] J. Silverman, The arithmetic of elliptic curves, Springer-Verlag Graduate Texts in Mathematics 106 (Springer-Verlag, Berlin, Heidelberg, New York, 1986).

[13] J. Tate, 'Algorithms for determining the type of a singular type in an elliptic pencil', in Modular Functions of One Variable IV, Lecture Notes in Mathematics 476 (Springer-Verlag, Berlin, Heidelberg, New York, 1972), pp. 33-52.

[14] J. Tunnell, 'A classical diophantine problem and modular forms of weight $3 / 2$ ', Invent. Math. 72 (1983), 323-334.

University of Szczecin

Institute of Mathematics

ul. Wielkopolska 15

70-451 Szczecin

Poland

e-mail: dabrowsk@sus.univ.szczecin.pl 